\title{
Tension Free Mesh Repair is an Effective Treatment of Inguinal Hernia: An Observational Study
}

\author{
Haque $\mathrm{SS}^{1}$, Islam $\mathrm{MT}^{2}$, Haque $\mathrm{MN}^{3}$, Rahman $\mathrm{MM}^{4}$, Ahmed $\mathrm{A}^{5}$
}

\begin{abstract}
Inguinal hernias are common and the results of surgical repair are often satisfactory but recurrence rate are variable. To prevent recurrences prosthetic materials have been increasingly used in hernia repair. In this study Lichtenstein technique of tension free mesh repair for inguinal hernia done in 140 cases in the period from July 2010 to June 2012 and outcome measured in terms of early and late morbidity especially recurrences. Seroma developed in 08 patients (5.6\%) and haematoma and transient testicular swelling developed in 06 patients (4.2\%) and 15 patients $(10.6 \%)$ respectively. Only 2 patients $(1.4 \%)$ developed wound infection and 01 patients $(.71 \%)$ developed recurrences of hernia. Post operative neuralgia developed in 05 cases (3.5\%). During follow up period no mesh rejection and discharging wound sinus related to presence of foreign body is observed in the study. So Lichtenstein tension free repair of inguinal hernia is safe and effective method where recurrence rate is reasonably low.
\end{abstract}

\section{Introduction}

Inguinal hernia, regardless of type is one of the most common diseases that a surgeon has to manage ${ }^{1}$. Mesh hernioplasty has gained widespread acceptance due to its superior outcome in terms of reduced recurrence rates which are in the range of $1-2 \% \%^{2,3,12}$. Improved surgical technique and a better understanding of the anatomy and physiology of the inguinal canal have significantly improved outcomes for many patients. Lichtenstein presented his open mesh repair technique for inguinal hernia in $1986^{4,14}$. Tension free mesh repair is nevertheless associated with complications such as foreign body reaction, pain, sinus formation, mesh migration etc. Meshes used are typically made from

1. Corresponding Author:

Dr. Sheikh Sayidul Haque FCPS, FRCS

Associate Professor of Surgery

Khulnaa Medical College, Khulna

E-mail: drhaque27@yahoo.com

2. Dr. Md. Tarikul Islam FCPS, MS

Assistant Professor of Plastic Surgery

Khulna Medical College, Khulna

3. Dr. Md. Nazmul Haque FCPS, MS

Junior Consultant of Urology

Shaheed Sheikh Abu Naser Specialized Hospital, Khulna

4. Dr. Md. Mustafizur Rahman

Assistant Registrar of surgery

Khulna Medical College, Khulna

5. Dr. Arif Ahmed

Assistant Registrar of surgery

Khulna Medical College, Khulna polypropylene or polyester. Various studies have suggested that low density and larger pore size may lead to decreased inflammatory response and less contracture, because it forms a thinner scar net $t^{5,6,15}$. This decreased inflammatory response may help improved outcome ${ }^{7}$. The aim of the study was to evaluate our experience of postoperative complications both early and late in a series of inguinal hernia repair by using prolene mesh in Lichtenstein technique.

\section{Materials And Method}

We performed these operations in different Govt. teaching and private hospitals in 140 patients in the period from July 2010 to June 2012. In this study, indirect inguinal hernias above the age of 40 years and direct and recurrent hernias irrespective of ages were included. Most of the predisposing risk factors were treated logically. Operations were done in Lichtenstein technique. We recorded the postoperative recovery and complications and reviewed the patients on 6 weeks and 6 months. Thereafter the patients were advised to contact on demand basis.

Most of the operations were done under spinal anaesthesia and few were done under epidural anaesthesia. Skin is prepared in usual way. Prophylactic antibiotics were given in each case before incision. In indirect hernia, sac is dissected up to deep inguinal ring and ligated in its neck and distal portion is excised. In large direct hernia, contents were imbricated with non-absorbable suture materials.

The Lichtenstein method employed a sheet of polypropylene mesh covering the posterior wall of inguinal canal extending for security, over adjacent structures, with a hole to transmit the cord. The overall dimension of the mesh is of $6 / 11 \mathrm{~cm}$. To accommodate this, external oblique apponeurosis is separated from the deeper layers superiorly and medially and from the muscular part of the internal oblique laterally to create an adequate pocket to receive the mesh. The lower medial corner of the mesh is constructed slightly rounded and upper medial corner rather more so. The mesh is incised from its lateral margin, cutting one third of distance from lower edge. The cut is extended approximately half the length of the mesh, depending upon the size of the patient.

The apex of the mesh is sutured to the pubic tubercle using a 3-0 prolene suture. The same continuous suture is used to fix the lower boarder of mesh to the free edge of inguinal ligament which is extended upto just medial to the anteriorsuperior iliac spine. Interrupted prolene sutures were used to approximate the cut edges of the mesh together around the spermatic cord. The infero-medial corner is attached well overlapping the pubic tubercle. The mesh is anchored 
to the conjoined tendon by interuppted prolene sutures no 3-0 ensuring good area of overlap medially, superiorly and laterally with good suture line inferiorly. The fibrosis induced by prolene mesh produce a sound result. The cord is replaced in the inguinal canal. Meticulous haemostasis ensured. External oblique appneurosis and skin were closed in layers.

In perioperative care prophylactic antibiotics were continued for 5 post operative days. Postoperative pains were managed mostly by NSAIDS. Patients were mobilized within first 24 hours of operation. The usual duration of hospital stay were 3-5 days

\section{Results}

A total number of 140 patients were treated by tension free mesh repair by Lichtenstein technique in the study period.Table-1 showing 86 patients $(61.0 \%)$ were indirect inguinal hernia, 45 patients $(32.0 \%)$ were of direct variety and remaining 09 patients $(06.4 \%)$ were recurrent inguinal hernia. $81,(57.5 \%)$ patients were right sided, 44 patients $(31.2 \%)$ were left sided and remaining 15 patients $(10.7 \%)$ were of bilateral.

Table-1: Type and side of hernia.

\begin{tabular}{lll}
\hline \multirow{3}{*}{ Side of hernia } & Right & n $(\%)$ \\
& Left & $44(57.5 \%)$ \\
Type of hernia & Bilateral & $15(10.7 \%)$ \\
& Indirect & $86(61.0 \%)$ \\
& Direct & $45(32.0 \%)$ \\
& Recurrent & $09(.06 .4 \%)$ \\
\hline
\end{tabular}

Complications in the early postoperative period were measured in terms of seroma, haematoma, transient testicular swelling and wound infection-shown in table-2. 08 patients $(5.6 \%)$ developed seroma and 06 patients $(4.2 \%)$ developed haematoma. Out of 06 patients only two required drainage and two other patients of seroma required repeated aspiration before finally resolved. Only 02 patients $(1.4 \%)$ developed wound infection which were treated by antibiotics and dressing. 15 patients $(10.6 \%)$ developed transient testicular swelling which settled spontaneously.

Table-2: Early outcome following operation.

\begin{tabular}{llll}
\hline Complications & Number (\%) & Measures taken & Outcome \\
\hline 1. Seroma & $08(5.6 \%)$ & $\begin{array}{l}\text { Only two required } \\
\text { repeated aspiration } \\
\text { Two patients required } \\
\text { drainage of haematoma }\end{array}$ & Resolved \\
2. Haematoma & $06(4.2 \%)$ & Scrotal elevation & Resolved \\
$\begin{array}{llll}\text { 3. Transient } \\
\text { testicular } \\
\text { swelling }\end{array}$ & $15(10.6 \%)$ & Dressing done and & Controlled \\
$\begin{array}{l}\text { 4. Wound } \\
\text { infection }\end{array}$ & $02(1.4 \%)$ & antibiotics given & \\
\hline
\end{tabular}

The patients in the study group were followed up postoperatively and reviewed on 6th week and on 6th month postoperatively. Thereafter the patients attended in the follow up clinic on demand basis. Follow up report are shown in the table-3. During the follow up period no mesh rejection and discharging wound sinus related to presence of foreign body is observed. One $(.72 \%)$ patient developed recurrences of hernias which were detected in the follow up period and treated as recurrent inguinal hernia. Postoperative neuralgia developed in 5 cases $(3.5 \%)$, which were managed by simple analgesics like NSAID and nortryptyline-most of the patients were settled.

Table-3: Follow up report.

\begin{tabular}{|c|c|c|c|}
\hline Complication & Number $(\%)$ & Measures taken & Outcome \\
\hline $\begin{array}{l}\text { 1. Postoperative } \\
\text { neuralgia }\end{array}$ & $05(3.5 \%)$ & $\begin{array}{l}\text { NSAID \& } \\
\text { Nortryptyline }\end{array}$ & Satisfactory \\
\hline 2. Recurrences & $01(.71 \%)$ & $\begin{array}{l}\text { Treated as } \\
\text { recurrent hernia }\end{array}$ & Resolved \\
\hline $\begin{array}{l}\text { 3. Chronic } \\
\text { discharging } \\
\text { wound sinus }\end{array}$ & Nil & & \\
\hline 4. Mesh rejection & Nil & & \\
\hline
\end{tabular}

\section{Discussion}

Tension free mesh repair of inguinal hernia was originally popularized by Lichtenstein in 1989 and it is most commonly performed operations for inguinal hernia now a days ${ }^{8,11}$. The description of Lichtenstein tension free mesh repair has opened a new era in groin hernia repair ${ }^{9,13}$. This method is very simple, effective, and is associated with very low recurrence rates (ranging from $0-2 \%$ in the literature) and can be performed under local or regional anaesthesia $^{16,17}$. As it is tension free, it causes minimal postoperative pain ${ }^{10,13}$. The technique used in this study is practical for our surgeons because there is virtually no learning curve. In the word of Amid "the open tension free repair is a typical example of 'see one, do one, teach one" ${ }^{\prime 18,19}$. For these important advantages, it is currently the preferred method for plastic reconstruction of inguinal hernia.

Closing the defect (in direct hernia) or narrowing it (in indirect hernia) is a crucial step in preventing recurrences. However the posterior wall tends to be weakened and breached again by persistent predisposing factors. In addition, wider defect in posterior wall than simple hernia demand to reinforce the posterior wall with mesh which has got obvious improvement over traditional surface repair ${ }^{19}$. Its effectiveness is demonstrated in this study in terms of rare recurrence rate.

The mesh is constructed of high-porosity polypropylene which provides a larger surface area for effective tissue in growth and fibrosis ${ }^{11}$. The properties of an ideal mesh are inertness, resistance to infection, molecular permeability pliability, transparency, mechanical integrity and biocompatibility ${ }^{13}$. Absorbable mesh does not remain long enough in the wound for adequate collagen to be deposited, while multifilament mesh can harbor bacteria ${ }^{17}$. Monofilament mesh is the most popular presently in use with various types of polypropylene having different characteristic advantages ${ }^{17}$. 
To reduce the recurrences the mesh should extent $2-4 \mathrm{~cm}$ beyond the boundary of Hesselbachs' triangle ${ }^{20}$. The position of mesh beneath the apponeurosis of the external oblique result in the intra abdominal pressure working in favour of the repair, since the external oblique apponeurosis keeps the mesh tightly in place by acting as a external support when intra abdominal pressure rises ${ }^{13}$. This method is very simple and effective. An extremely low recurrence rate $(0-.7 \%)$ has been reported in many groups of surgeons ${ }^{16}$. All these factors have encouraged our surgeons as a choice of method of hernia repair.

The early and late outcome following Lichtenstein tensionfree inguinal hernia repair is quite satisfactory. Pain is well controlled in this study. Early post operative complications were minor. There was no significant evidence of infection in the study and recurrence rate approximately $0.7 \%$ which is extremely low. More-over for rapid post operative recovery and rapid return to unrestricted activities, this technique is preferred method of inguinal hernia repair. Considering our experience it is acceptable, practical, effective and safe for the patient

\section{References}

1. Bowen JR, Thompson WR, Dorman BA, Soderbery $\mathrm{CH}$, Shahimian TK: Change in the management of adult groin hernia. Am. J.Surg. 1977,135:564-9

2. The EU hernia trialist collaboration. Repair of groin hernia with synthetic mesh. Meta-analysis of radomized controlled trials. Ann.Sur. 2002;235:322-32

3. olzheimer RG(2007) Low recurrence rate in hernia repair-result in 300 patients with open mesh repair of primary inguinal hernia. Eur. J. Med.Res 2007;12:1-5

4. O dwyer PJ. Kingsnorth AN. Molloy RG. Small PK. Lamers B:Randomized clinical trials assessing impact of a lightweight Or heavyweight mesh on chronic pain after inguinal hernia repair. J. Sur.2005;92:166-70

5. Welty G, Klinge U, Klossterhalfen B, Kasper KR, SShumpelick KV. Functional impairment and complaints following incisional hernia repair with with different polypropylene meshes. hernia 2001;5:142-7

6. Klunge U, Klosterhalfen B, Berkenhauer V, Junge k, Conje J.Shampelick KV Impact of polymer pore size on the interface scar formation in a rat model. J. Surg. Res.2002;103:208-14

7. Bringeman S, Wellert S, Osterberg J, Smedberg S, Grandlaud H, Heikman TJ, Three years results of a randomized clinical trial of lightweight or standard polypropylene mesh in Lichtenstein repair of primary inguinal hernia:Br. j. Surg. 2000;93:1056-9
8. Lichtenstein IL, Shulman AG, Amid PK, Montllor MM, The tension free hernioplasty. AM. J Sueg, 1989,157:188-93

9. Bassini E, Sulla cura radicle dell ernia inguinale. Arch Soc Ital chir 1887;4:380-88

10. Debord JR, THe historical development of prosthetics in hernia surgery. Surg, Clin, North. Am. 1998,78:9731006

11. Chandiramani Vinod A. Katara Avinash N, Pangya Shetali M, Nair Nita S, Prolene hernia system in the tension free repair of primary inguinal hernia.Indian $\mathrm{J}$. Surg. 2003; 65:488-91.

12. Lt.Col.Shyam S, Jaisal, Brig Rajan Chowdry Maj amit agarwal, Chronic groin pain following Lichtenstein mesh hernioplasty for inguinal hernia. Is it a myth? Indian J Surg. 2009;71:84-88

13. George HS, Loannis H, Christos N, Nikolaos K, Alexios S. Constantinos A et al. Open tension free repair of inguinal hernias: the Lichtenstein technique. BMC J Surg 2001,1:3

14. Schumpelic V Klinge U, The properties and clinical effect of various types of mesh used in hernia repair. Association of Great Britain and Ireland (yearbook)2001

15. Nadim K, Muhammad N, Adil B, Haris H, Early outcome of Lichtenstein technique of tension free open mesh repair for inguinal hernia. J Ayub.Med Coll.Attottabad. 2008;20:29-33

16. Kurzer M. BelshamPA, Kark Ke. The Lichtenstein repair .Surg. Clin. North. Am. 1998;78:1025-46

17. Goldstein HS, Selecting the right mesh. Hernia 1999;3:23-26

18. Fitzgibbon RJ, Amid PK, Corbitt JD, McKernan Jb, Nyhus LM, Pyne JH. Symposium: Operative repair of inguinal hernia-part-2 Contemporary surgery 1998;110

19. Nathaniel I. Usoro:Early outcome of inguinal hernia repair using ultrapore mesh in University of Calabar Teaching Hospital, Nigeria. The internrt Jr of third wprld Medi. ISSN:1539-4646

20. Amid PK, Shulman AG, lichtenstein IL. Open tension free repair of inguinal hernia: the lichtenstein technique. Eur J Surg. 1996;162;447-53 